\title{
Outbreak of Highly Pathogenic Avian Influenza in Commercial Poultry Farms, Kwahu-West Municipality, Ghana
}

\author{
Francis Nuvey ${ }^{1}$, Basil Kaburi ${ }^{1}$, Esther Dsani ${ }^{1}$, Pascal Mwin ${ }^{1}$, Eli Dzandu ${ }^{1}$, Timothy \\ Wekura $^{1}$, Joseph $\mathrm{Abu}^{2}$, Donne Ameme ${ }^{1}$, and Ernest Kenu ${ }^{1}$ \\ ${ }^{1}$ University of Ghana \\ ${ }^{2}$ Ghana Ministry of Food and Agriculture
}

August 23, 2020

\begin{abstract}
Background Avian Influenza is a highly contagious viral infection that mainly affects domesticated birds. On June 21, 2018, the Kwahu-West Municipal Veterinary Office was notified of sudden deaths of birds on a poultry farm. Seven days later, a second farm sited 100 meters from the index case-farm also recorded bird deaths. We investigated to confirm the causative agent, identify its source, and implement control measures. Methods We conducted a survey of six poultry farms and human contacts of affected farms in Nkawkaw. A suspected case-farm of HPAI was any farm in Nkawkaw with sudden death of bird(s), with or without clinical signs of HPAI from June 1 to July 10, 2018. Six bird carcasses were collected on affected farms for laboratory analysis. We interviewed owners of affected farms and assessed the farm environments, as well as clinical status of human contacts. Results A total 2,280 birds were affected on two adjacent farms. HPAI-H5N1 was confirmed for all samples investigated. The index case-farm with 1,438 birds, reported 30 bird deaths on June 16, 2018, 24 hours after receiving a consignment of birds from an HPAI-H5N1 confirmed farm in a neighboring region of Ghana. The second case-farm recorded bird deaths 7 days later. Both farms operated aluminum sheet-roofed metallic mesh pens without fencing. Beddings in pens were not regularly changed. Four poultry farms located within an $6 \mathrm{~km}$ radius of the affected farms did not report sick or dead birds. All twenty-three human contacts were asymptomatic of flu, after 14-days follow-up. Conclusions This HPAI-H5N1 outbreak was likely imported through the introduction of birds from a neighboring region due to lax livestock movement regulations, and poor biosecurity measures on farms. Disinfection and depopulation exercises effectively controlled the outbreak. We recommend strict implementation of biosafety measures on farms and at entry points in the district
\end{abstract}

Title: Outbreak of Highly Pathogenic Avian Influenza in Commercial Poultry Farms, Kwahu-West Municipality, Ghana

\section{Running Title: Avian Influenza in Commercial Poultry Farms, Ghana}

Francis Sena Nuvey ${ }^{1,2 *}$, Basil Benduri Kaburi ${ }^{1,2}$, Esther Dsani ${ }^{1,3}$, Pascal Kingsley Mwin ${ }^{1,2}$, Eli Dzandu ${ }^{1,2}$, Timothy Wekura ${ }^{1}$, Joseph $\mathrm{Abu}^{3}$, Donne Kofi Ameme ${ }^{1,2}$, Ernest Kenu ${ }^{1,2}$

${ }^{1}$ Ghana Field Epidemiology and Laboratory Training Program, Department of Epidemiology and Disease Control, School of Public Health, University of Ghana, Accra, Ghana, ${ }^{2}$ Ghana Health Service, Ministry of Health, Accra, Ghana, ${ }^{\mathbf{3}}$ Veterinary Service Department, Accra, Ghana

*Corresponding author

Email: fsnuvey@gmail.com 


\section{Abstract}

\section{Background}

Avian Influenza is a highly contagious viral infection that mainly affects domesticated birds. On June 21, 2018, the Kwahu-West Municipal Veterinary Office was notified of sudden deaths of birds on a poultry farm. Seven days later, a second farm sited 100 meters from the index case-farm also recorded bird deaths. We investigated to confirm the causative agent, identify its source, and implement control measures.

\section{Methods}

We conducted a survey of six poultry farms and human contacts of affected farms in Nkawkaw. A suspected case-farm of HPAI was any farm in Nkawkaw with sudden death of bird(s), with or without clinical signs of HPAI from June 1 to July 10, 2018. Six bird carcasses were collected on affected farms for laboratory analysis. We interviewed owners of affected farms and assessed the farm environments, as well as clinical status of human contacts.

\section{Results}

A total 2,280 birds were affected on two adjacent farms. HPAI-H5N1 was confirmed for all samples investigated. The index case-farm with 1,438 birds, reported 30 bird deaths on June 16, 2018, 24 hours after receiving a consignment of birds from an HPAI-H5N1 confirmed farm in a neighboring region of Ghana. The second case-farm recorded bird deaths 7 days later. Both farms operated aluminum sheet-roofed metallic mesh pens without fencing. Beddings in pens were not regularly changed. Four poultry farms located within an $6 \mathrm{~km}$ radius of the affected farms did not report sick or dead birds. All twenty-three human contacts were asymptomatic of flu, after 14-days follow-up.

\section{Conclusions}

This HPAI-H5N1 outbreak was likely imported through the introduction of birds from a neighboring region due to lax livestock movement regulations, and poor biosecurity measures on farms. Disinfection and depopulation exercises effectively controlled the outbreak. We recommend strict implementation of biosafety measures on farms and at entry points in the district.

Keywords: Avian influenza, Poultry, Outbreak, Biosecurity

\section{Background}

Avian influenza is a viral disease that infects bird species, with varying levels of severity, depending on the virus strain involved. The viruses with $\mathrm{H} 5$ or $\mathrm{H} 7$ surface proteins cause the most severe form of avian influenza, and are described as the highly pathogenic avian influenza (HPAI) [1]. Wild birds are the common vectors of the avian influenza viruses. The virus is however highly contagious among domesticated birds, including chickens, ducks, and turkeys. The infection occurs when a bird directly comes in contact with other infected birds, or indirectly through contact with contaminated water, feed, and surfaces. The HPAI may cause disease affecting multiple internal organs, with a 48-hour mortality rate ranging between $90-100 \%$ [2].

According to the World Health Organization (WHO) and International Organization for Animal Health (OIE), human infections with avian influenza viruses have been sporadically reported in all the regions in the world, majority of which originated from Asia [3,4]. However, person-to-person transmission of avian influenza viruses is rarely reported. The risk of avian influenza infection of humans remains high if biosafety measures are neglected, and is mainly related to outbreaks among poultry [5]. Most of the avian influenza outbreaks in humans result from close contact with sick domesticated birds [6]. The ability of avian influenza viruses to cross the species barrier is because they are constantly evolving through mutation and genetic 
re-assortment leading to the emergence of new subtypes, thereby posing significant threat to both animal and human health [7].

The avian influenza viruses that have crossed the species barrier to infect humans include A(H5N1), A(H5N6), $\mathrm{A}(\mathrm{H} 7 \mathrm{~N} 4), \mathrm{A}(\mathrm{H} 7 \mathrm{~N} 9)$, and A(H9N2). Since its emergence in China in 2013, the outbreaks of avian influenza virus $\mathrm{A}(\mathrm{H} 7 \mathrm{~N} 9)$ has infected more humans (excess of 1500 human cases) than any of the other types. However, avian influenza $\mathrm{A}(\mathrm{H} 5 \mathrm{~N} 1)$ has caused the largest number of cases of severe disease and death in humans $[2,4]$. According to the WHO, more than 860 human cases of avian influenza $\mathrm{A}(\mathrm{H} 5 \mathrm{~N} 1)$ virus were reported from 17 countries globally, with more than 50\% case fatality rate between January 2003 and December 2019 [4].

In many African countries, outbreaks of HPAI A(H5N1), $\mathrm{A}(\mathrm{H} 5 \mathrm{~N} 2)$ and $\mathrm{A}(\mathrm{H} 5 \mathrm{~N} 8)$ in poultry have been confirmed [3]. The first of the infection in Africa occurred in Nigeria in 2006, which subsequently spread quickly to at least 17 other countries on the continent by 2017 [7,8]. Only three African countries including Djibouti, Nigeria, and Egypt reported confirmed human cases of avian influenza (H5N1) on the continent since its first confirmation in 2006 [7]. The first human case of HPAI (H5N1) was confirmed on March 20, 2006 in Egypt. By November 2010, more than 100 human cases were confirmed in Egypt with majority of the confirmed cases linked to contact with poultry kept in backyard farms [9]. Globally, Egypt recorded the highest number of 346 confirmed human cases by the end of 2015 [7].

Ghana reported its first confirmed HPAI A(H5N1) outbreak in poultry in 2007 in three regions including Greater Accra, Volta, and Brong Ahafo Regions. Until 2015, no outbreaks of HPAI were reported, with the Greater Accra Region first confirming cases of H5N1 in April 2015. Subsequently, five of the sixteen regions in Ghana, also recorded confirmed cases by June the same year, affecting both commercial and freerange poultry farms and leading to losses of approximately 100,000 birds [10]. Between 2017 and 2018, the A(H9N2) strain was identified in outbreaks in poultry in the Ashanti and Brong-Ahafo Regions of Ghana [11]. The spread of avian influenza in Ghana has mainly been attributed to poor biosafety practices on farms and poor regulation of movement of live poultry [10]. No human cases of HPAI have been confirmed in Ghana since its introduction in 2007 [7].

On June 21, 2018, a farm owner notified the Veterinary Office in the Kwahu West Municipality of the death of birds on his small-scale commercial poultry farm at Nkawkaw. A different small-scale commercial farm located about 100 meters from the index-case farm also started recording deaths of birds 7 days later. Upon visiting the farms, the Municipal Veterinary Officers suspected a highly pathogenic avian influenza outbreak. Based on their notification, the Eastern Regional Health Directorate through the Municipal Health Management Team (MHMT), constituted a team to respond to the outbreak. We investigated the outbreak to confirm the causative agent, identify its source, assess risk factors for spread, and implement control measures.

\section{Methods}

\section{Outbreak setting}

The outbreak investigation was conducted from June 25 to 30, 2018 in Nkawkaw, capital of the Kwahu West Municipality in the Eastern Region of Ghana (Figure 1). This was the first reported avian influenza outbreak in the municipality. The municipality is one of the twenty-six (26) districts in the Eastern Region of Ghana [12]. The projected population for Kwahu West Municipality is about 115000 in 2018. About half of the population live in the rural areas [13]. Nearly $44 \%$ of households in the municipality are engaged in agricultural activities including crop farming, livestock rearing and fish farming.

Livestock rearing is the second most important agricultural activity accounting for about $25 \%$ of all agricultural activities. About $61 \%$ of livestock kept are birds with $95 \%$ being chicken [12]. The birds are mainly raised on a small-scale backyard basis with few commercial farms. Farm capacities range between 100 and 5,000 birds. About 47,000 commercial layers and 70,000 domestic or rural birds were raised in the district 
in 2018. There were three live bird markets located within the Nkawkaw Central Market near the roadside, where trading of birds and eggs is mainly done [14].

Only one veterinary clinic provides veterinary services to livestock farmers in the municipality. Fifty (50) health facilities provide human healthcare services in the municipality. These include three Hospitals, nine Health Centers, two maternity homes, and thirty-six Community-based Health Planning and Services (CHPS) facilities. There is no routine surveillance for Influenza-like illnesses in the Kwahu West Municipality. The only influenza sentinel facility in the Eastern Region is located in the Koforidua Regional Hospital, which is about 86 kilometers from Nkawkaw.

\section{Figure 1: Map of Ghana showing the outbreak setting (colored sea blue)}

\section{Study design}

We conducted a survey involving interviews with poultry farmers, human contacts of affected farms and veterinary personnel. We also conducted records review and environmental assessment. The interviews with the farmers were conducted using a structured questionnaire detailing farm demographic characteristics, flock history, farm biosecurity, farm management practices, contacts information and other risk factors to HPAI (S1 File). We actively searched for case farms within an 6 kilometer radius of the index-case farm and identified six farms for assessment. We defined a suspected HPAI case-farm as a poultry farm (domestic or commercial) in Nkawkaw with sudden death of birds, with or without clinical signs of HPAI on from June 1 to July 10, 2018. Six (6) farm owners/managers were interviewed. This includes the managers of the two affected and four other unaffected farms. All twenty-three contacts of the case farms were followed up daily for 14 days. The contacts were interviewed with a structured questionnaire to assess for any clinical manifestations of influenza. We defined a primary human contact as any person who has had physical contact with the case farms, and/or the birds or eggs of case farms from June 15 to 262018 . A secondary human contact was defined as any person exposed by physical contact with a primary contact.

We reviewed the surveillance data of influenza-like illnesses (ILI) and other upper respiratory tract infections in the Kwahu West Municipality for the period of 2017 and the five months preceding the outbreak in 2018 retrieved from the District Health Information Management System (DHIMS-2) database. We conducted environmental assessment of the immediate vicinity of both farms to identify risk factors to the spread of the avian influenza infection including the presence of wild birds and water bodies close to pens as well as the biosecurity measures practiced on the farms.

\section{Laboratory investigations}

Six whole bird carcasses were collected from the two affected farms. The fresh carcasses were transported in appropriate double-layered plastic bags inside a leak-proof plastic container to the Accra Veterinary laboratory within four hours of collection. In this Biosafety Level 3 (BS-3) laboratory, viral ribonucleic acid (RNA) was extracted from cloacal and oropharyngeal swabs taken from the carcasses and diagnosed using Reverse Transcription Polymerase Chain Reaction (RT-PCR). The samples were tested for influenza viruses adhering to all standard protocols [15]. Appropriate personal protective equipment were donned in the handling of the specimen.

\section{Data analysis}

We performed descriptive analysis of the outbreak data by person, place and time. We calculated overall mortality rate and farm specific mortality rates. Continuous variables including age of human contacts were expressed with appropriate measures of central tendency and dispersion. The results were presented as frequencies and relative frequencies in tables and graphs. We drew a column graph to describe the magnitude and the course of the death of birds. We used quantum geographic information system tools [16] to map the poultry farms in the outbreak setting. Data was analyzed using Stata version 15.0. 


\section{Coordination}

The response to the outbreak was organized through multi-sectoral collaboration in a One Health approach. Field epidemiologists from the Ghana Field Epidemiology and Laboratory Training Programme supported the Municipal Health Management Team to respond to the outbreak. The team constituted by the Kwahu West Municipal Health Management Team included staff of the Ghana Health Service, Veterinary Services Department, and Municipal Assembly. The Municipal Fire Service Department, National Disaster Management Organization, and Ministry of Food and Agriculture supported the investigation team in the response to the outbreak.

\section{Ethical considerations}

The investigation was considered a response to a public health emergency by the Ghana Health Service and Veterinary Services Department and therefore did not receive formal review by Ethical Review Committees. We sought and obtained permission from the Eastern Regional Health Directorate, Eastern Regional Veterinary Services Directorate, Kwahu West Municipal Assembly, Kwahu West Municipal Veterinary Department and Kwahu West Municipal Health Management Team before commencement of the investigation. All respondents provided consent and were assured of confidentiality. We anonymized all information collected on the affected farms and human contacts and stored them securely as confidential records.

\section{Results}

\section{Outbreak characteristics}

A total of 2,188 birds were present at the time of the outbreak on the two affected farms (Table 1). The first Case farm had 1,438 birds while Case farm 2 had 750 chickens at the time of the outbreak. In all, 189 dead birds were reported. The overall mortality rate of birds was 9 per 100 birds. The farm specific mortality rates were 13 per 100 birds for Case farm 1 and 2 per 100 birds for Case farm 2.

Table: Distribution of exposed poultry birds by farms in Nkwakaw, Kwahu West Municipality

\begin{tabular}{lll}
\hline Bird type & Case farm $\mathbf{1}$ & Case farm 2 \\
\hline Chicken & 1400 & 750 \\
Ducks & 38 & 0 \\
Total & 1438 & 750 \\
\hline
\end{tabular}

The interviews with affected farm owners revealed that the owner of Case farm 1 bought 650 live birds (layers) from a farm in a neighboring region in three consignments of 200, 250 and 200, and transported these birds without any inspection. The birds bought were kept in a separate pen from the farmer's existing flock. After the owner of the first affected farm (Case farm 1) observed thirty (30) dead and ninety (90) sick birds in his farm 24 hours after taking delivery of the last consignment of birds bought, he returned these birds back to the originating farm for replacement, also without any inspections. Although the birds were replaced, more deaths of birds were reported among the birds that were bought. The farmer sold some of the birds that looked healthy in the live bird market in the district. Subsequently, more deaths of birds were reported among his existing flock and, on a nearby farm. The farmers eventually reported the bird deaths to the Municipal Veterinary Office six (6) days later after they tried self-treating without success. Figure 2 illustrates the key events in the outbreak.

Figure 2: Flow diagram of key events that occurred between June 15 and 262018 during the Highly Pathogenic Avian Influenza Outbreak, Nkwakaw, Kwahu West Municipality, June 
The graph (Figure 3 ) below illustrates an incremental growth in the number of dead birds recorded on the affected farms, after the initial multiple deaths (30 birds) reported on Case farm 1, until the depopulation exercise conducted on June 26.

Figure 3: Number of bird deaths by day of death during the Highly Pathogenic Avian Influenza outbreak in Nkwakaw, Kwahu West Municipality, June 2018

We found an intersection in the two case-farms within a $200 \mathrm{~m}$ buffer area plotted around the case and non-case farms (Figure 4 ). S2 Figure illustrates an 6 kilometer radius about the Case farm 1 and all other bird farms within the radius.

Figure 4: Distribution of Highly Pathogenic Avian Influenza infected and non-infected farms about the index case farm

\section{Characteristics of contacts}

In all, 23 human contacts were identified including 20 primary contacts $(87 \%)$ and 3 secondary (13\%) contacts. About $53 \%(12 / 23)$ of the contacts were males. The median age of the contacts was 25 years (range $=5$ to 64 years), with majority $65 \%(15 / 23)$ being resident at Amanfrom, an area close to the affected farms. All contacts were exposed between June 15 and 26, 2018 inclusive. None of the 23 contacts identified were symptomatic for influenza during the outbreak period and after follow up.

\section{Laboratory results}

Reverse transcription polymerase chain reaction (RT-PCR) results confirmed highly pathogenic avian influenza (HPAI-H5N1) for all the six whole bird carcasses tested using universal primers for avian influenza viruses $\mathrm{H} 5$ and $\mathrm{H} 7$.

\section{Findings from environmental assessment}

Both case farms were sited in a peri-urban area, but none of them was fenced. The two affected farms are about 100 meters apart. Case farm 1 had three pens used for poultry with sizes; 40 by 40 feet, 30 by 40 feet, and 25 by 20 feet. The farm also housed goats, sheep, and rabbits. The structure of the pen comprised concrete foundation with metallic mesh for walls and roofed with aluminum sheets. Each pen had feeding and water troughs. The water troughs were improvised from cooking oil gallons that were cut. Two of the pens lie directly on the side of a pathway that residents living behind the farm, use. There was a well dug on the compound from which water is drawn for domestic use and watering the animals. There was a small pen in-between the three pens where sick birds were kept for observation. There was a stream with a wooden bridge approximately 20 meters northwards from the Case farm 1. Wild birds were seen perching on the pens. The pens housing goats, sheep and rabbits were directly adjacent the pens with birds. The litter produced were stored in sacks by the side of the pens. The beddings were also not regularly changed.

Case farm 2 had two pens used for raising only poultry birds. The pens measure about 25 by 30 feet and 30 by 40 feet respectively. The pens had concrete floors and foundation. Metallic mesh was used to cover the open area from the foundation up to the roofing. The pens were roofed with aluminum sheets. Behind one of the pens was a manhole. No wild birds were seen on Case farm 2. The maintenance of the pens was similar to what was observed on Case farm 1. Human movements were reported between the two case farms. 


\section{Discussion}

The highly pathogenic avian influenza (HPAI) virus generally causes severe outbreaks in poultry population. However, occasionally HPAI infect humans exposed to infected poultry. The HPAI-H5N1 outbreak confirmed in this study have been implicated in previous outbreaks in many African countries including Nigeria, Egypt, Côte d'Ivoire, Burkina Faso, Niger and Cameroun [10,17]. In Cameroun, two strains of HPAI; H5N1 and H5N8 were isolated in outbreaks that occurred on commercial farms in 2016 affecting birds with mortality rates ranging between $8 \%$ and 96\%. In Ghana, previous outbreaks of HPAI (H5N1 and H9N2) have been reported in many regions since the first outbreak in 2007 [10,11]. The circulating H5N1 and H9N2 strains in Ghana have been reported to be homologous to that observed in Nigeria, Burkina Faso and other West African countries, suggesting a potential cross-border contamination of bird species [10,11]. The low mortality rate; $9 \%$ and $13 \%$, observed on Case farms 2 and 1 respectively in the current study, are similar to those previously reported in the West Africa region [18]. This may be explained by the similarity in the strains identified in the region.

Our study revealed concerning findings of how birds were moved between farms without inspection by regulatory bodies at the entry points of the district. Even more concerning was how one of the affected farmers moved dead and sick birds back to the farm (source) in the neighboring region for replacement without detection. These lapses contributed to the importation and subsequent spread of the HPAI outbreak in the affected farms. Similar links with poor regulation, were shown in previous HPAI outbreaks $[9,10,17,19]$. The key sources of the transmissions of HPAI previously reported includes unregulated movements of poultry and eggs dealers from one farm, market, or town to another without appropriate preventive care as well as poor biosecurity measures on farms and live poultry markets [17]. In Nigeria, similar biosecurity non-compliances have been implicated in H5N1 and H5N8 outbreaks occurring between 2014 and 2017 [7]. Similar lapses in biosecurity measures were implicated in HPAI outbreaks globally especially in Europe and Asia [20-23]. The poor regulation of movement of animals have a potential to affect trade negatively in the future.

Owing to the difficulties in restricting the movement of poultry in many settings, some control strategies implemented in previous HPAI outbreaks in Egypt include mass vaccination, surveillance and preemptive culling of infected birds [9]. In Ghana, control measures utilized in previous HPAI outbreaks involved depopulation of all birds on affected farms, disinfection of affected farms, and restricted movement of poultry and poultry products after which, active influenza surveillance is initiated among birds, domestic poultry, and the human population [10]. These measures were equally effective in the current outbreak response. The depopulation of all birds on affected farms, disinfection of farms and live bird market, and ban on sale and movement of live birds during the current outbreak response, contributed to a successful control of the outbreak. Similar control measures have been used in other settings with success $[9,21]$. The collaborative efforts of both human and animal health personnel through a One Health approach as well as the legislation and enforcement by the municipal assembly through the municipal coordinating council also contributed largely to containment and prevention of further spread of the infection in the present outbreak.

Although human cases of HPAI have been reported in 15 different countries following outbreaks in birds including in Hong Kong [21], Thailand [24], Turkey [25], Egypt [26] and Cameroon [17], none of the human contacts in our study developed flu-like symptoms after two maximum incubation periods follow-up. Almost all of the human cases reported in previous avian influenza outbreaks were caused by zoonotic transmission from poultry and disproportionately affects women and their children. This is because women and children were observed to be more exposed when nursing birds raised in backyard farms compared to adult males who are less involved on the backyard farms [9]. Transmission generally depends on every country's own dynamics associated with raising poultry and other intrinsic immunologic susceptibility to the infection. Nevertheless, transmission of HPAI from poultry to humans continues to be rare, despite frequent and widespread close contact between humans and affected poultry [27]. However, our study could not assess how and why the human contacts were asymptomatic after follow-up.

The effects of avian influenza outbreaks are often devastating for farmers. Generally, the economic conse- 
quences of these outbreaks are severe, since it results in culling of all affected birds and their eggs [28]. To alleviate the impact of losses suffered on farmers and encourage farmers to report bird deaths, payment of financial compensation to farmers is common in some settings including in Egypt [9] and the United States of America [29]. Although compensation was not paid to the affected farmers in the present outbreak, the farmers were educated and counselled to prevent future outbreaks.

\section{Public Health Actions}

The affected farmers were informed of laboratory confirmation of HPAI-H5 on Saturday June, 232018 . The farmers were educated on the essential biosecurity measures to adopt to mitigate future occurrences. They were sensitized on the need for regular hand washing with soap and running water after attending to the poultry. The health facilities within the municipality were also placed on high alert for influenza-like illnesses and any unusual events.

The Municipal Assembly initiated a ban on the sale and movement of birds from affected farms. Similarly, a ban on the sale of live birds at the Nkawkaw market was also instituted. A depopulation exercise was conducted on June 26, 2018. A pit of about 3 meters high was dug with an excavator and the birds transported in a container to the pit together with all the eggs found on the farms. Carbon dioxide foam concentrate was sprayed on the birds in the pit before they were covered with a soil layer of about 2 meters. Disinfection of the live bird market was conducted on June 27, while the infected farms were disinfected on June 30, 2018. The farms were cleared of remaining litters and beddings and the decontamination of the pens done using Izal solution.

\section{Conclusions}

An outbreak of HPAI subtype H5N1 occurred on two adjacent farms located in Nkawkaw in the Kwahu West Municipality between June 16 and 26 2018. The source of the outbreak was probably birds purchased from a commercial farm in Boankra in the Ashanti Region of Ghana by the owner of Case farm 1 on June 15 2018. The outbreak was confined to the two farms in the affected municipality. Rapid response to the outbreak involving depopulation and disinfection of affected farms and live bird market, ban on sale of live birds and eggs in the markets and health education to the community helped in controlling the outbreak and preventing more cases. The implementation of strict monitoring and certification of livestock before, during and after movement in the municipality, sensitization of the security services on biosafety measures at various entry and exit points in the municipality, and regular and routine inspection of poultry farms by veterinary personnel are justified measures to prevent future occurrences.

\section{Declarations}

\section{Acknowledgments}

We acknowledge support received from the staff of the Accra Veterinary Laboratory, Ghana Health Service, Veterinary Services Department, and Kwahu West Municipal Assembly who contributed to a successful resolution of the outbreak. In addition, we would like to thank the technical assistance provided by the Afrique One African Science Partnership for Intervention Research Excellence, Ghana Field Epidemiology and Laboratory Programme and Field Epidemiology and Laboratory Network of Ghana.

\section{Competing interests}

DKA and EK declare that they are editorial board members of BMC Public Health. All other authors have no competing interests. 


\section{Funding}

None to declare.

\section{Author Contributions}

Conceived and designed the study: FSN BBK ED PKM ED TW DKA EK. Conducted the investigation: FSN BBK ED PKM ED TW JA. Analyzed the data: FSN ED PKM ED. Contributed materials/analysis tools: BBK DKA EK. Wrote the paper: FSN. Edited the paper FSN BBK ED PKM ED TW DKA EK.

\section{Data Availability Statement}

All relevant data are within the manuscript and its Supplemental Materials files.

\section{References}

1. WHO. Influenza virus infections in humans. Geneva; 2014.

2. CDC. Avian Influenza (Bird Flu). Atlanta; 2006. (January).

3. OIE. OIE Situation Report for Highly Pathogenic Avian Influenza. Paris; 2018.

4. WHO. Human infection with avian influenza A (H5) viruses. Geneva: WHO Western Pacific Region; 2019.

5. WHO. WHO public health research agenda for influenza: 2017 update. Geneva: World Health Organization; 2017.

6. CFSPH. Highly Pathogenic Avian Influenza. Iowa; 2016.

7. Fasanmi OG, Odetokun IA, Balogun FA, Fasina FO. Public health concerns of highly pathogenic avian influenza H5N1 endemicity in Africa. Vet World. 2017;10.

8. Cattoli G, Monne I, Fusaro A, Joannis TM, Lombin LH, Aly MM, et al. Highly pathogenic avian influenza virus subtype $\mathrm{H} 5 \mathrm{~N} 1$ in Africa: A comprehensive phylogenetic analysis and molecular characterization of isolates. PLoS One. 2009;4(3):1-9.

9. Abdelwhab EM, Hafez HM. An overview of the epidemic of highly pathogenic H5N1 avian influenza virus in Egypt: epidemiology and control challenges. Epidermiol Infect. 2011;139:647-57.

10. Asante IA, Bertram S, Awuni J, Commey ANO, Aniwa B, Ampofo WK, et al. Highly pathogenic avian influenza A(H5N1) virus among poultry, Ghana, 2015. Emerg Infect Dis. 2016;22(12):2209-11.

11. Awuni JA, Bianco A, Dogbey OJ, Fusaro A, Yingar DT, Salviato A, et al. Avian influenza H9N2 subtype in Ghana: virus characterization and evidence of co-infection. Avian Pathol. 2019;48(5):470-6.

12. Ghana Statistical Service. District analytical report: Kwahu west. Accra; 2014.

13. Ghana Statistical Service. Population projections by districts, 2015 - 2020. Accra; 2016.

14. VSD. 2018 Annual Report, Eastern Region. Koforidua; 2019.

15. OIE. Highly Pathogenic Avian Influenza. In: OIE Manual of Diagnostic Test and Vaccines for Terrestrial Animals. Paris: OIE; 2004.

16. QGIS Development Team. QGIS Geographic Information System [Internet]. Open Source Geospatial Foundation; 2013. Available from: http://qgis.osgeo.org

17. Kouam MK, Tchouankui HN, Ngapagna AN. Epidemiological Features of Highly Pathogenic Avian Influenza in Cameroon. Vet Med Int. 2019;1-6. 
18. FAO. Wild Birds and Avian Influenza: an introduction to applied field research and disease sampling techniques. In: Whitworth D, Newman SH, Mundkur T, Harris. P, editors. FAO Animal Production and Health Manual [Internet]. 5th ed. Rome: FAO; 2007. Available from: www.fao.org/avianflu

19. Olsen S, Laosiritaworn Y, Pattanasin S, Prapasiri P, Dowell SF. Poultry-handling Practices during Outbreak, Thailand Avian Influenza. Emerg Infect Dis. 2005;11(10):1601-3.

20. De Zwart O, Veldhuijzen IK, Elam G, Aro AR, Abraham T, Bishop GD, et al. Avian Influenza risk perception, Europe and Asia. Emerg Infect Dis [Internet]. 2007;13. Available from: https://doi.org/10.3201/eid1302.060303

21. Koch G, Elbers ARW. Outdoor ranging of poultry: A major risk factor for the introduction and development of High-Pathogenecity Avian Influenza. NJAS - Wageningen J Life Sci [Internet]. 2006;54(2):179-94. Available from: http://dx.doi.org/10.1016/S1573-5214(06)80021-7

22. Singer RS, Hofacre CL. Potential Impacts of Antibiotic Use in Poultry Production. Avian Dis. 2006;50(2):161-72.

23. Suarez DL, Senne DA, Banks J, Brown IH, Essen SC, Lee CW, et al. Recombination Resulting in Virulence Shift in Avian Influenza Outbreak, Chile. Emerg Infect Dis. 2004;10(4):693-9.

24. Areechokchai D, Jiraphongsa C, Laosiritaworn, Y Hanshaoworakul W, O'Reilly M. Investigation of avian infl uenza (H5N1) outbreak in humans - Thailand, 2004. MMWR. 2006;55(SUP01):3-6.

25. Sarikaya O, Erbaydar T. Avian influenza outbreak in Turkey through health personnel's views: A qualitative study. BMC Public Health. 2007;7(330):1-11.

26. Kandeel A, Manoncourt S, Abd E, Ahmed AM, El-refaie S, Essmat H, et al. Zoonotic Transmission of Avian Influenza Virus (H5N1), Egypt, 2006 - 2009. Emerg Infect Dis. 2010;16(3):2006-9.

27. Kerkhove MD Van. Brief literature review for the WHO global influenza research agenda - highly pathogenic avian influenza H5N1 risk in humans. Influ J. 2013;7(Suppl. 2):26-33.

28. Chatziprodromidou IP, Arvanitidou M, Guitian J, Apostolou T, Vantarakis G, Vantarakis A. Global avian influenza outbreaks 2010 - 2016: a systematic review of their distribution, avian species and virus subtype. Syst Rev. 2018;7(17).

29. Greene JL. Update on the Highly-Pathogenic Avian Influenza Outbreak of 2014-2015. 2015.

\section{Supplemental Materials}

S1 File: Data collection tools used in outbreak investigation

S2 Figure: Map showing an 6 kilometer radius about the Case farm 1 and the bird farms within the radius. 

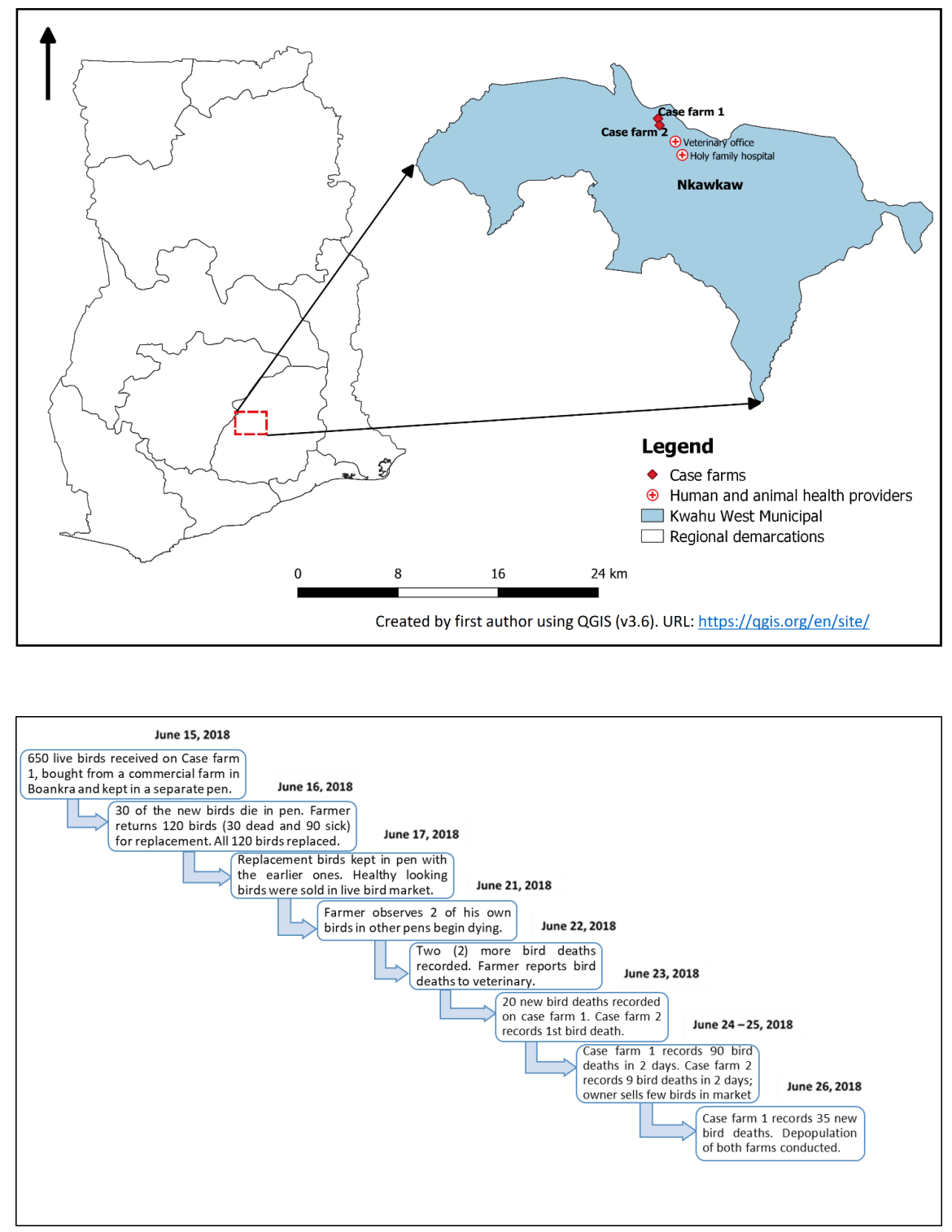

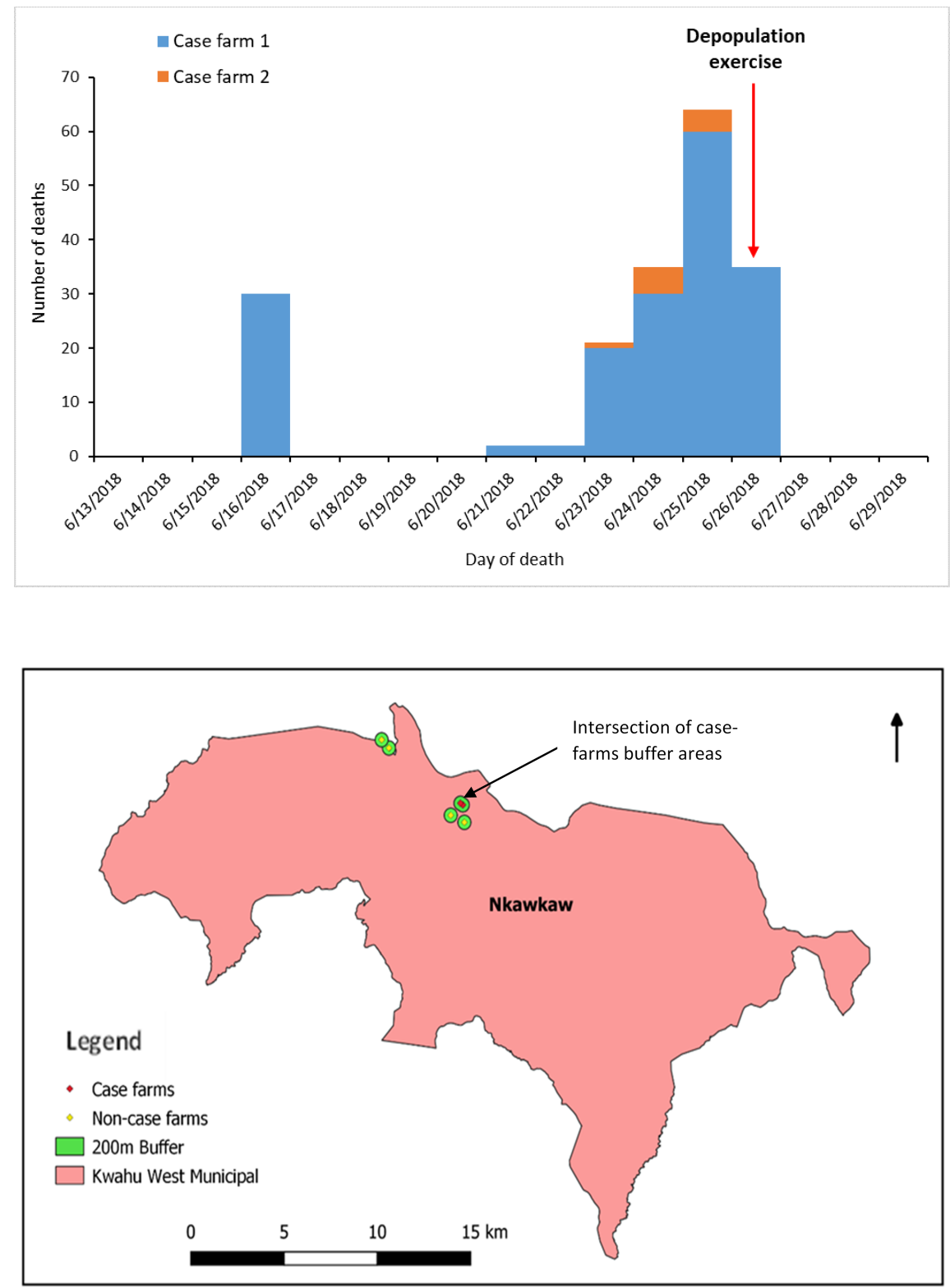

\section{Hosted file}

Table.docx available at https://authorea.com/users/352164/articles/476519-outbreak-ofhighly-pathogenic-avian-influenza-in-commercial-poultry-farms-kwahu-west-municipalityghana 